Témoigner Témoigner. Entre histoire et mémoire

Getuigen Revue pluridisciplinaire de la Fondation Auschwitz

$125 \mid 2017$

Histoire et mémoire de la persécution des

homosexuel-le's par les nazis

\title{
La répression de l'homosexualité en France entre 1940 et 1945
}

De bestraffing van homoseksualiteit in Frankrijk tussen 1940 en 1945

Jean-Luc Schwab

(2) OpenEdition

Journals

Édition électronique

URL : https://journals.openedition.org/temoigner/6470

DOI : $10.4000 /$ temoigner.6470

ISSN : 2506-6390

Traduction(s) :

De bestraffing van homoseksualiteit in Frankrijk tussen 1940 en 1945 - URL : https://

journals.openedition.org/temoigner/6500 [nl]

Éditeur :

Éditions du Centre d'études et de documentation Mémoire d'Auschwitz, Éditions Kimé

Édition imprimée

Date de publication : 1 octobre 2017

Pagination : 95-107

ISBN : 978-2-930953-01-4

ISSN : 2031-4183

Référence électronique

Jean-Luc Schwab, «La répression de l'homosexualité en France entre 1940 et 1945 », Témoigner. Entre histoire et mémoire [En ligne], 125 | 2017, mis en ligne le 24 décembre 2021, consulté le 03 février 2022. URL : http://journals.openedition.org/temoigner/6470; DOI : https://doi.org/10.4000/temoigner.6470 


\section{La répression de l'homosexualité en France entre 1940 et 1945}

$\rightarrow$ Jean-Luc Schwab

Jean-Luc Schwab

étudie la répression

de l'homosexualité en

France durant la Seconde

Guerre mondiale. II

poursuit ses recherches en

préparation d'une thèse

sur les déportés en lien

avec l'homosexualité au

camp de concentration de

Natzweiler.

Il est par ailleurs l'auteur

d'une biographie sur

Rudolf Brazda (1913-2011),

dernier survivant connu de

la déportation pour motif

d'homosexualité (Itinéraire

d'un Triangle Rose, Paris,

Florent Massot, 2010/J'ai

Lu, 2013).

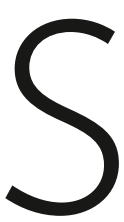

'intéresser aux persécutions qui ont frappé spécifiquement les individus accusés d'homosexualité en France durant la Seconde Guerre mondiale revient à traiter d'un sujet étonnement polémique aujourd'hui encore. Périodiquement s'affrontent dans l'espace public et médiatique partisans et opposants d'une réalité historique qui n'est plus contestable, mais dont toutes les nuances restent mal connues. En effet, si la France a été touchée à des degrés divers, ce phénomène est d'abord le fait du régime nazi qui, au gré de sa politique expansionniste puis guerrière, va appliquer, hors des limites territoriales de l’Allemagne d'après le Traité de Versailles, des mesures qui lui sont propres. En Allemagne, la répression initialement d'ordre légal repose sur l'article 175 du Code pénal - remontant à l'époque impériale - dont les dispositions ont été renforcées par le régime hitlérien en 1935. À cette loi se sont greffées des pratiques coercitives, principalement policières, et cet arsenal punitif est, pour ainsi dire, «exporté » à la suite des annexions et conquêtes armées de l'Allemagne (comme l'évoque aussi Florence Tamagne dans un article de ce numéro). Toutefois, l'application qu'en font les nazis est fonction des territoires qui passent sous domination allemande et de la politique qu'ils y mènent.

Si la France est également concernée, elle ne l'est pas de façon uniforme partout sur son sol. Car après l'armistice du 22 juin 1940, la France vaincue par les forces allemandes est partagée en trois zones principales, à l'exclusion toutefois de la Corse et de quelques territoires limitrophes, respectivement occupés par l'Italie fasciste dans le sud-est ou rattachés au nord au Commandement militaire allemand de Bruxelles :

- une zone occupée, placée sous administration allemande, englobant grossièrement les départements au nord de la Loire, ainsi qu'une large bande littorale atlantique jusqu’à la frontière espagnole ;

- les deux départements alsaciens et la Moselle, annexés de fait;

- la portion sud restante, siège du gouvernement de Vichy, dite « zone non occupée » ou «zone libre » jusqu’à sa prise en main préventive par les autorités allemandes en novembre 1942, à la suite du débarquement allié en Afrique du Nord. 
La répression de
lhomosexualité en France entre 1940 et 1945

Or les mesures de lutte contre l'homosexualité prises en France entre 1940 et 1945, c'est-à-dire entre l'invasion du territoire français par les armées allemandes et la libération complète du pays, doivent être appréhendées à laune de cette division territoriale imposée par l'envahisseur nazi. Comme on le verra ici, la répression de l'homosexualité connait des formes variées qui sont à mettre en relation avec la manière dont les autorités allemandes ou françaises perçoivent l'homosexualitédans chacune de ces grandes zones territoriales. Comme le montre également la situation particulière de l'Alsace annexé, cette région fournit une clé de compréh essentiele du phénomène en France, tant pour l'histoire que pour sa memoire. Ca l'étude historique permet aussi une approche critique de la mêmoire collective et militante pour ce qui touche au souvenir des personnes persécutées en France sur accusation d'homosexualité durant cette période.

\section{AUX PRÉMICES D'UNE RECHERCHE}

Après avoir considéré cette dimension territoriale, aborder de façon historicisante la répression de l'homosexualité en France occupée ou annexée requiert une approche circonspecte quant à son traitement jusque dans les années 2000. Auparavant les principaux auteurs d'écrits françis sur le sujet, souvent des militants (Guy vant,les ch (Guy Hocquenghem, Jean Boisson ou Jean Le Bitoux notamment), ne pouvaient guère se targuer d'avoir recueilli un grand nombre de témoignages de victimes, encore moins d'avoir procédé à des investigations détaillées dans les fonds d'archives contemporains conservés tant en France qu'en Allemagne. Faute d'informations fiables, ces militants de lémancipation homosexuelle, longtemps les seuls à revendiquer l'existence d'une telle persécution en France, n'ont pas toujours su apporter une réponse convaincante et fondée à leurs contradicteurs. C'est encore le cas de nos jours, les affirmations de certains militants LGBT (lesbiennes, gaies, bi, trans') se caractérisant souvent par l'approximation et les propos rapportés, tant en ce qui concerne les chiffres que les sources disponibles (Bourgi, 2015, p. 356-363) tandis que la persécution se voit réduite aux emblématiques déportés au triangle rose. que la pétude des Or l'étude des sources archivistique montre que la pratique répressive de pant allemand, lorsqu'elle se traduit par une privation de liberte, privilegie d'autre structures de détention que les seuls canps de concentration. Il convient donc de traiter avec prudence ce qui a pu en être écrit et dit par les acteurs de la mémoire (pouvoirs publics, institutions et associations mémorielles, anciens déportés pour d'autres motifs) et les militants LGBT, dès lors que le propos ne s'appuie pas sur des sources attestées.

Car les premières recherches valables sont somme toute très récentes en France, quelque peu plus anciennes en Allemagne pour ce qui touche aux zones an parle Reich par le peich allo Arna Bolligy, cherch à la Fondation pour la débute un recensement des Français pour qui une accusation d'homosexualité a pu conduire tantôt en camp de concentration, tantôt dans d’autres lieux de détention en Allemagne ou sur le sol français. Sa source principale se trouve d'abord dans les archives du ministère de la Défense, compétent pour traiter les demandes effectuées après-guerre par les victimes ou leur ayants droit en vue d'obtenir un titre de déporté ou d’interné. Ses contacts dans les mémoriaux allemands des camps de concentration ainsi que des copies de documents du Service international de recherches (SIR) de Bad Arosen lui permettentégolement d'établir une première recheme liste de Français explicitement signalés comme «homosexuels» dans les camps de concentration, particulièrement celui de Natzweiler (nom germanisé du village alsacien où est initialement implanté ce camp, désigné localement sous l'appellation de son lieu-dit d'implantation, au «Struthof»). Ses travaux sont les seuls dont on dispose pour ce qui est de cette répression par le régime nazi en France, hors zone annexée. Pour celle-ci et l'Alsace en particulier, je me baserai essentiellement sur mon étude des archives départementales du Haut-Rhin et du Bas-Rhin, de l'état civil, ainsi que diverses sources inédites provenant des institutions pénitentiaires et psychiatriques que conservent les établissements qui remplissent ces fonctions aujourd'hui encore (Schwab, 2015). D’autres fonds ont également été consultés en Allemagne tout particulièrement ceux du SIR ainique danscertains départements relevant de lancienne zone non occupée. Enfin, pour la Moselle, les renseignements rassemblés par l'historien Cédric Neveu ont permis de compléter le tableau pour la
zone annexée (Neveu, 2012).

\section{LE CONTEXTE FRANÇAIS}

Avec le Code pénal de 1791 et l’abandon du crime de sodomie par le pouvoir issu de la Révolution, la loi française ne condamnait plus les pratiques homosexuelles consenties entre hommes (Lever, 1985). Encore au début du XXe siècle, l'homosexualité n'est pas condamnée en tant que telle. Des poursuites peuvent toutefois être engéés lorsue léćéments à charge sont suffisonts pour constituer un

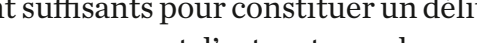
d'outrage public à la pudeur, ce chef d'inculpation recouvrant d'autres types de pra-
tiques qui ne se restreignent pas à l'homosexualité.

Après la débâcle de 1940, le gouvernement français est dirigé depuis Vichy (zone non occupée) parle Maréchal Pétain qui bénéficie des pleins pouvoirs depuis le 10juillet. Malgré la tutelle allemande, le «gouvernement de Vichy » conserve sa faculté de légiférer et son autorité sur les préfets qui le représentent dans les départements et les régions, y compris dans la zone nord occupée. Le 6 août 1942, dans un esprit de reconquête morale, caractéristique de sa « révolution nationale», Vichy édicte une loi rendant passible d'amende pécuniaire et de réclusion tout acte d'homosexulité

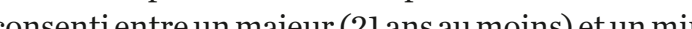

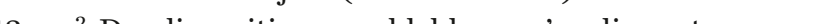
loi crée de facto une discrimination sur lâge du consentement sexuel pour les actes

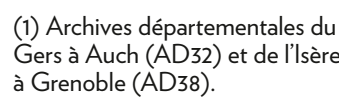
(2) Journal Officiel, Loi in 744 du
6 a aout 1942 modifiant larticle 334 du Code pénal. 
La répression de "homosexualité en France
entre 1940 et 1945 entre 1940
(suite)

d'homosexualité. Autre nouveauté, elle s'applique désormais à l'homosexualité tant masculine que féminine. Quelques exemples de procédures débouchant sur des condamnations à des peines de réclusion ont pu être retrouvés isolément en zone sud (Boninchi, 2005, p. 161-193). On pourrait penser que cette législation aurait eu un impact déterminant dans le pays, mais elle ne s'accompagnera pas d'une répression systématique à grande échelle. Des statistiques globales annuelles n'existent qu’à compter de 1945, année où le ministère de la Justice dénombrait en tout et pour tout 22 inculpations pour udélitd'homosexulité Mas l'élémentbien plus déterminant pour les années a venir est l'introduction insidieuse dans le droit français de la notion d'acte «contre nature» et la stigmatisation de l'homosexualité à qui elle ouvre la voie (Jackson, 2009, p. 45-55). Les années 1940 à 1945, marquées par l'Occupation, la perte de souveraineté ainsi qu'un gouvernement de collaboration avec l'ennem dans un contexte de guerre à léchelle continentale, constituent de ce point de vue une période charnière pour la France. Elles méritent qu’on s'attarde au traitement réservé à l'homosexualité dans chacune des grandes zones distinguées plus haut :

RÉPRESSION DE L'HOMOSEXUALITÉ ET

INTERNEMENT ADMINISTRATIF EN ZONE SUD

Quoique ses effets initiaux soient difficilement quantifiables, la loi instaurée par Vichy semble avoir une portée très limitée entre sa mise en œuvre et la libération progressive du territoire français durant la seconde moitié de 1944, même dans la zone sud où siégeait le gouvernement du Maréchal Pétain. Aujourd'hui cependant, un autre type de mesures coercitives prises à l'encontre d'hommes accusés d'homosexualité émerge peu à peu de l'étude des archives de l'internement administratif, avec des exemples remontant pour certains à la seconde moitié de l'année 1940, soit presque deux ans avant l'introduction de ladite loi. Le principe de l'internement administratif s'enracine dans le décret du 18 novembre 1939 qui vise à empêcher de nuire «les individus dangereux pour la défense nationale ou pour la sécurité publique et dispose de leuréloignement du lieu de résidence, voire del'sucurité

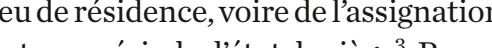
à rèsider dans un lieu désigné à cet effet pendantune période d'etat de siège ${ }^{3}$. Revue et adaptée par Vichy, cette mesure est initialement prononcée par les préfets qui, sur proposition de la police, décident de l'internement en Centre de séjour surveillé (CSS) pour une durée déterminée, l'éloignement avec assignation à résidence demeurant une alternative.

La consultation détaillée des fonds correspondants aux archives départementales du Gers à Auch fait apparaître sept cas qui renvoient à l'homosexualité des mis en cause. Il ressort une tendance des policiers enquêteurs à traiter plus sévèrement certains troubles à l'ordre public - surtout l'intempérance et l'ivresse sur la voie publique -lorsque l'homosexulité est mentionnée com la voie publique 1943) qu’un policier gersois signale comme indésirable en mai 1941, et à l'encontre de qui «il serait utile

de prendre un arrêté d'internement dans un centre de séjour surveillé», l'individu étan «connu pour se livrer des mœurs spéciales et a la boisson. á láfoisson. L'arrêté préfectoral émis pe après - et 13 jours seulement après son arivé dans ce département suite à son expulsion d'Alsace annexée pour homosexualité - précise en outre qu'il est de «moralité douteuse» et serait «susceptible de se livrer à n'importe que ivrer à n'inporte quel endroit à ses mour particulières.» ${ }^{4}$ D'abor

envoye au CSS de Siste-

ron dans les Basses-Alpes, il y reste près d'un an et demi, jusqu’à son transfert au CSS de Fort-Barraux dans l'Isère à la mi-novembre 1942. À l'époque déjà, sa santé fragile inspirait quelques craintes à l'administration. L'ironie du sort veut que la décision commuant sa détention administrative en assignation à résidence dans le Gers arrive quelques jours seulement après son décès, survenu le $1^{\mathrm{er}}$ février 1943 à l'infirmerie du CSS $^{5}$.

Trois autres cas d'internements administratifs prononcés par le préfet des Alpes-Maritimes à Nice, pratiquement à l'autre extrémité de la zone non occupée, ont également pu être repérés. Là aussi, les internés passent par différents lieux de ont également pu être repérés. Là aussi, les internés passent par differents lieux de détention avant le CSS de Sisteron puis celui de Fort-Barraux. Dans le dossier de
l'un d'eux, Henri P. (1920-1975), interné par arrêté préfectoral du 31 octobre 1940, l'un d'eux, Henri P. (1920-1975), interne par arrêté préfectoral du 31 octobre 1940, on note l'appréciation du chef du CSS de Fort-Barraux à son sujet : «Il s’agit d'un homosexuel, qui déclare ne pas avoir travailléjusqu’à ce jour et avoir tiré ses subsides en fréquentant des invertis.» Pour finir, l'individu «n'envisageant pas de retravailler, sa libération obtenue», un avis défavorable est émis, avis suivi par les autorités décisionnaires ${ }^{6}$. Le 22 juin 1944, lui et les deux autres concernés sont du convoi parti de Grenoble à destination du camp de concentration de Buchenwald où ils vont être astreints au travail forcé au profit de l'économie de guerre allemande. Des trois, c'est le seul survivant recensé. Il est mort célibataire dans sa ville natale, trente ans après sa libération d'Allemagne. de la plaque commémoran les déportés pour motif d'homosexualite Natzweiler (Alsace), 25 septembre 2010.
(4) AD 32 , Auch, dossiers
individuels, cotes 1 W1 158 et 159 (5) $\mathrm{AD} 38$, Grenoble, dossiers (6) AD38, Grenoble, ditto 
La répression de
Phomosexualité en France entre 1940 et 1945
(suite)

Ici aussi, l'accusation d'homosexualité - formulée très explicitement, contrairement au camouflage langagier utilisé par l'administration dans le premier exemple est un facteur aggravant, voire un motif suffisant pour justifier de l'internemen administratif sous couvert de protection de l'ordre public. À une époque où la loi de Vichy ne réprime pas encore certaines relations homosexuelles consenties, peut-on dès lors parler d'une politique concertée de répression de l'homosexualité au travers de ces mesures d'internement? Seule l'étude des archives d'autres départements françis de la zone non occupée permettra à terme d'établir s'ils agissit tendance plus profonde. Pour autant, cette découverte ouvre une piste de recherche jusque-là insoupçonnée.

\section{RÉPRESSION DE LA COLLUSION SEXUELLE AVEC L'OCCUPANT ET DE LOISIVETÉ EN ZONE NORD}

Dans cette large portion du territoire français que les conditions de l'armistice du 22 juin 1940 placent sous l'autorité allemande, le gouvernement français provisoire, ensuite investi des pleins pouvoirs et installé à Vichy, garde son autorité sur l'administration d'Etat bien que l'occupant y applique ses propres règles en matière de maintien de l'ordre. Malgré la tutelle allemande, les appareils policier et judiciaire préexistants sont maintenus, sans que la législation allemande ne se substitue au corpus de lois françaises, y compris en matière d'homosexualité ou d'outrage public à la pudeur. En théorie, cela signifierait que jusqưà l'entrée en vigueur de là loi d’août 1942, rien ne change en ce qui concerne le traitement légal des pratiques homosexuelles, tant qu'elles font intervenir des individus consentants, d'âge légal uffisant, dans un cadre strictemen privé. Pour autant, les dernières - Détail avant sa pose 25 septembre 2010 .

\section{ALA MÉMOIRE}

DES VICTIMES

DE LA BARBARIE NAZIE;

\section{DEPORTEEES POUR MOTIF}

\section{DHOMOSEXUALITE} magne depuis la zone nord : 10 en camps de concentration 12 dans le système carcéral (Boulligny, 2010, p. 51-72). Deux typologies sont discernables :

Tout d'abord, et majoritairement, le reproche que leur font le autorités militaires allemande d'avoir eu, ou voulu avoir, des rapd’avoir én, on voulu avoir, des rapports débauchés avec les personnels des forces d’occupation en France.
Dans ce cas, des juridictions militai- res allemandes ont prononcé des peines de prison sur la base du droit allemand (article 175) à l'encontre d'accusés ou coaccusés français pour qui la réclusion va s'effectuer dans des prisons allemandes. Dans d'autres cas, plus anecdotiques, une accusation d'espionnage sur fond d'homosexualité est brandie contre les concernés. Comme dans ce dossier commun à deux individus sur qui les enquêteurs français déclarent dans un rapport du 15 décembre 1942 : «Des renseignements recueillis sur les motifs de son arrestation, il résulte qu'il semble s'agir d'une affaire de mœurs, d'escroquerie et d'espionnage traitée par les Autorités d'occupation. ${ }^{7}$ 'aflaire dans d'escrolle l' deux laquelle l'un d'eux est accusé d'avoir entretenu une liaison avec un officier allemand a des suites fâcheuses pour ces deux amis que rapprochait uniquement la fréquentation des mêmes lieux de sociabilité homosexuelle encore présents dans la capitale. Leur internement, débuté au mois de novembre 1941, se poursuit dans différents sites de la région parisienne et les mène en janvier 1944 au camp de concentration de Buchenwald. Ils n'y sont pas désignés comme homosexuels, mais comme «politiques français» et portent le triangle rouge correspondant, marqué d'un F. À une exception près, c'est le cas de tous les déportés concentrationnaires partis des zones occupée ou non occupée et dont la détention auparavant avait pour origine une affaire d'homosexualité. Le seul déporté au «triangle rose» depuis la France occupée se trouve d'ailleurs être un soldat allemand, arrêté fin juin 1942 par les occupés to des autorités militaires allemandes, condamné à 15 mois de prison pour « débauche entre hommes », transféré dans diverses prisons allemandes, puis aux camps
concentration de Natzweiler-Struthof et Dachau où il est désigné « $\$ 175 »,{ }^{8}$

Par ailleurs, on ne connait actuellement qu’un seul Français déporté depuis la zone nord, Gilbert Doublet (1909-1945), pour qui le lien avec Ihomosexualite est formellement établi par une mention explicite sur une liste de transfert de France vers l'Allemagne9. Malgré cela, il est lui aussi désigné comme «politique français» dans les camps de concentration de Buchenwald et Mittelbau-Dora et l'annexe d'Ellrich où il meurt le 21 février 1945. Sans qu'on n'en sache beaucoup plus sur les circonstances de son arrestation, il ferait potentiellement partie d'une autre catégorie, moins fournie dans les chiffres mais pas moins inintéressante.

Cette seconde catégorie qui relie le reste des individus touchés tiendrait plus de la lutte contre «l'oisiveté», c'est-à-dire la répression d'individus ne justifiant pas d'un travail régulier, en particulier après la mise en place début 1943 du Service du Travail obligatoire (STO) au bénéfice de l'Allemagne. La relation causale est souvent difficile à établir avec certitude, mais les rapports de police français fournissent des faisceaux de présomption non négligeables sur les interpelés qui ont par la suite été remis aux autorités allemandes. C'est par exemple le cas pour l'acteur de théâtre et cinéma Hugues Lambert (1908-1945) ou encore l’artiste Jean Giobellina (1918-1943). Selon les témoignages, le Selon létablissement connu pour recevoir une clientèle homosexuelle ${ }^{10}$. Quant au second, il es présenté comme un «pédéraste notoire, fréquentant les bals clandestins, durant les
(7) Archives de la Préfecture de Henri T. [1906-1974] \& Lucien

(8) ITS Arolsen, Josef Bellarmin
D. (1903-1969) - Questionnaire US pour détenus des camps
de concentration $-1.1 .6 .2-$ Document 10018040 . (9) ITS Arolsen, Liste alphabétique de détenus KL Buchenwald le le 27.06.1943 1.1.5. - Document 53444380 (10) DAVCC, Caen, Dossier- 
La répression de
lhomosexualité en France Thomosexualité en
entre 1940 et 1945
(suite)

années d'occupation. C'est pour ces motifs [...] qu'il aurait été arrêté le 22 août 1943 probablement par des policiers français. $»^{11}$ Tous deux sont déportés comme «politiques» au camp de concentration de Buchenwald et meurent d'épuisement. Lambert le 7 mars 1945 au camp de Flossenbürg où il a été transféré, Giobellina au camp annexe de Dora, trois mois seulement après son arrestation à Paris.

Si l'on considère l'ensemble formé en France par les zones occupée et non occupée, les priorités en matière de luttecontrel'homosexulité ains que leurévolution leur évolution entre 1940 et 1945 dépendent assez marginalement de la législation du gouvernemen de Vichy ou de ses pratiques policieres. La répression hors territoires annexés est donc limitée, même de la part de l'occupant allemand dont les autorités militaires ne sanctionnent les Français que lorsque des membres de ses forces armées sont impliqués. Dans cette logique, c'est bien plus la préservation du peuple allemand que vise le régime nazi, également hors de ses frontières. Cela vaut aussi pour les populations considérées comme de sang allemand à qui le régime va appliquer les lois du Reich dans les territoires annexés, également en matière de lutte contre l'homosexualité.

\section{LALSACE ANNEXÉE DE FAIT AU PREMIER RANG DE LA RÉPRESSION}

Redécoupés et remodelés au cours du XIX siècle, les actuels départements alsaciens (Haut-Rhin, Bas-Rhin) et celui de la Moselle voisine forment une zone qui avait été cédée àl'Empire allemand par le traité de Francfort en 1871. Les états allemands, fraîchement fédérés en un nouvel empire sous l'égide de la Prusse, scellaient ains leur victoire sur la France de Napoléon III par une prise de guerre conséquente. Avec le Traité de Versailles, ces territoires repassent dans le giron français à l'issue de la Première Guerre mondiale, ayant ainsi connu une première période durant laquelle la « débauche contre nature entre hommes » était condamnable d'après les termes du Code pénal impérial (Hoffschildt, 2013). Fin juin 1940, ils sont derechef placés sous le joug allemand - unilatéralement cette fois, d’où cette désignation ltérieure a d'annexion defaitr-etvoient française à l'égard des pratiques homosexuelles masculines qui n’appellent pas la même réponse légale en France qu’en Allemagne.

Une fois l'envahisseur nazi installé en Alsace, la traque qu'il y mène contre l'homosexualité masculine a d'abord pour toile de fond l'épuration de la population de ses éléments indésirables : politiques, criminels et «asociaux». Moins de deux mois après l'invasion de la région par les troupes du Reich, on trouve déjà trace dans les archives d'hommes accusés de pratiques débauchées homosexuelles cícece expéditives prises par lappareil policier, en dehors de décisions de justice, comme le montrent diverses lappare poliverse noter n’est pas relâché faute d'éléments à charge suffisants. Soit il est expulsé, soit il est envoyé pour une durée limitée au camp de sécurité de Schirmeck-Vorbruck lorsque l'administration nazie estime qu'il est susceptible de s'améliorer par le travail forcé qui est le lot des internés de ce camp. Ce fut $a$ priori le cas parmi d’autres de Pierre Seel (1923-2005). Dans les cas plus bénins, un avertissement doublé d'une amende peut conclure la détention, comme pour cet habitant de Colmar qui, en mars 1941, au bout de 23 jours en prison et grâce au témoignage à décharge de son épouse, voit

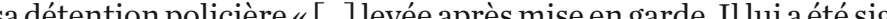
en garde. Illui a èté signifié qưen cas de récidive, des mesures policières de sécurité lui seraient appliquées.»"

Évoluant au gré de la mise au pas de l'Alsace par les nouveaux maîtres des lieux, la répression se judiciarise en février 1942 lorsque le Code pénal allemand supplante définitivement les dernières lois françaises encore en vigueur. Les bases légales étant officiellement jetées, le traitement judiciaire va s'imposer comme moyen de lutte prépondérant contre l'homosexualité masculine, avec à la clé une centaine de condamnations à des peines de réclusion. Entre 80 et 90 sont prononcées par les instances siégeant à Strasbourg, les autres à Colmar, Mulhouse ou Saverne. S’y rajoutent une vingtaine de verdicts contre des Alsaciens employés hors de la région, essentiellementen Moselle Sarre ou Pays de Badevoisins, rarement dans des juridicessentic tions plus lointaines. Les peines varient d'un mois a quatre ans de réclusion pour les actes d'homosexualité consentie, pouvant monter à cinqvoire huit ans de pénitencier pour les cas très lourds qui relèvent toutefois de l'abus sexuel sur mineurs plutôt que du simple acte consenti. Deux tiers des peines infligées le sont pour des durées d'incarcération supérieures à un an, la valeur médiane se situant autour d'un an et demi. Quelques jugements ont pu imposer une alternative à la détention pénale, notamment avec l'internement en établissement psychiatrique pour les personnes dont la responsabilité pénale est diminuée. Le dernier procès strasbourgeois pour délit d'homosexualité a lieu le 19 octobre 1944 et se solde par une condamnation à six mois de réclusion pour l’accusé.

Mais en plus des peines prononcées pour infraction aux dispositions des articles 175 et 175a (cas aggravés) d'autres mesures extrajudiciaires ont pu s'appliquer comme en Allemagne, notammentla Vorbeugungshaft ou «réclusion de prévention». Son but est de parer à léventualité d'une récidive ou d'isoler de la sociététout individu que la police estime représenter un danger persistant, quand bien même sa peine de prison a été purgée. À compter de 1942, ce type de détention s'effectue aussi au camp de sécurité de Schirmeck-Vorbruck, et non pas dans un camp de concentration comme cela se pratique déjà ailleurs en Allemagne. C'est là un particularisme observé en Alsace et qui concerne une vingtaine d'hommes, mis à l'épreuve, dont le cas doit en principe être réévalué 12 mois après leur arrivée au camp Dans les faits, ce déla '’ rive à n'a a partir de septembre 1944. Les concernés vont donc rester détenus
(12) Archives départementales
du Haut-Rhin, Colmar, 22AL3-8, du Haut-Rhin, Colmar, 22AL3-8,
Dossier individuel de Nicolas Dossier individuel de Nicolas
T. (1894-1983) - Traduction libre
de lauteur. 
La répression de
lhomosexualité en France lhomosexualité en
(nuite) 1940 et 1945

Enfin, le camp de concentration de Natzweiler, bien que d’abord réservé aux détenus déjà présents ailleurs dans le système concentrationnaire, reçoit lui auss quelques Alsaciens à partir de 1943. Ils viennent grossir les rangs de plus de 300 détenus en lien avec l'homosexualité, très majoritairement originaires du Reich, repérés dans le camp principal dès mai 1941, et plus marginalement dans ses annexes jusqưà la libération définitive de celles-ci en 1945.

Chiffrée et catégorisée selon les critères que le législateur français fixe en 1948 pour l'attribution d'un titre de déporté et les compensations afférentes, la répression pourlattribution d'un titre de déporté et les compensation

Quelque 150 hommes, très majoritairement alsaciens, sont assimilables à des déportés politiques (incarcérés plus de 3 mois dans le Reich et/ou les territoires annexés pour des motifs autres que de droit commun) : pour un peu plus des deux tiers, il s’agit d’individus condamnés à des peines de plus de six mois de réclusion par les tribunaux; les autres (incluant Pierre Seel) sont des détenus de sécurité qui ont passé plus de trois mois au camp de Schirmeck-Vorbruck, voire son site de repli en Allemagne.

Onze Alsaciens ont été déportés en camps de concentration, le premier dès novembre 1941 à Flossenbürg. Soit ils tombent sous le coup d'une mesure de réclusion de prévention prononcée hors d’Alsace, soit ils ont été transférés depuis un autre lieu (camp de Schirmeck ou hôpital psychiatrique) à la demande des autorités pour «être astreints au travail». Ils ne sont que cinq explicitement désignés comme homosexuels et donc porteurs du triangle rose (avec trois autres Mosellans, ils sont les seuls déportés français marqués de ce signe taxinomique). Les autres sont immatriculés comme "politiques alsaciens» ou «droit commun ».

Quelque 200 personnes sont directement touchées par une autre forme de répression non assimilée à de la déportation (incarcération de moins de 3 mois, expulsion maison de correction, placement en établissement psychiatrique en cas d'irresponsabilité pénale). On compte aussi quelques victimes collatérales chez les ćpouses ou les enfants qui suivent un mari ou un père expulsé.

Parmi les victimes directes (quelque 370 en l'état), on recense pour l'Alsace au moins 21 décès, majoritairement chez les détenus concentrationnaires ou en camps spéciaux (10); les expulsés (4 dont 3 en CSS); les détenus incarcérés en prisons (4) ou en hôpital psychiatrique (1). À noter les représailles spécifiques pou deux Allemands impliqués dans des affaires d'homosexualité : l'un est exécuté en vertu du décret secret sur le maintien de la pureté morale dans la SS et les forces de police ; l'autre, militaire allemand stationné en Alsace, meurt dans un bataillon disciplinaire dans lequel il a été rétrogradé On trouve en effet parmi les victimes une quarantaine d'étrangers, majoritairementdes residents réguliers en Alicte des avant-guerre, ou des Allemands établis en Alsace après l'annexion, voire en transit, comme les militaires $^{13}$.
En compilant ces données avec celles d'Arnaud Boulligny pour le reste de la France, on constate que l'Alsace annexée est à l'heure actuelle la région française la plus fortement touchée. Elle rassemble les trois quarts des quelque 500 personnes frappées par une forme de répression (incluant plus d'une centaine de Français poursuivis pour infraction aux articles 175 ou 175a, présents sur le sol allemand en tant que prisonniers de guerre, travailleurs volontaires ou contraints). Ce résultat pourrait ne pas être surprenant si l'on considère la spécificité de territoire annexé,

Ce département assez semblable au Bas-Rhin en termes de superficie et de population, est nettement moins touché. Si dès le début de la période d’annexion les expulsions y furent proportionnellement importantes, elles ont laissé moins de traces, aussi n'est-il pas possible d'établir d'ordre de grandeur pour ce qui est des homosexuels frappés d'une telle mesure. On dispose cependant de quelques données sur la répression judiciaire et le travail de dépouillement effectué par l'historien Cédric Neveu aux archives départementales laisse apparaittre une vingtaine d'hommes poursuivis et condamnés pénalement, dont plus d'une moitié d’Allemands. Parmi les Mosellans, parfois jugés pour d'autres délits de moralité en plus de l'accusation d'homosexualité, on recense en outre cinq déportés concentrationnaires dont un dhó immigré polonais. Ils sont d abord internés au KL Natzweiler, sauf un, envoyé directement a Dachau dans la «baraque des prêtres» (Schwab, 2015). Deux d'entre eux ne rentrent pas de déportation. Si l'apparente différence d'ampleur du phénomène entre la Moselle et l'Alsace pourrait tenir à des archives très fortement lacunaires, pour Cédric Neveu elle se justifierait davantage par le peu d'intérêt porté à cet aspect de la lutte contre les «asociaux» par le Gauleiter Bürckel et les autorités policières compétentes en Moselle (Neveu, 2012, p. 117-121).

\section{CONCLUSIONS}

Le morcèlement administratif et territorial pratiqué par l'envahisseur nazi entre 1940 et 1945 est essentiel pour appréhender la répression de l'homosexualité entre 1940 et 1945 est essentiel pour apprehender la repression de l'homosexualité en France durant ces années. Cest dans les territoires annexés et tout particulièrement en Alsace qu'elle est la plus féroce. En tout, près de 400 individus au moins y sont touchés par des sanctions dont le spectre s'étend de la détention arbitraire sans suites jusquàa'envoi en camp de concentration. Cette dernière option ne représente qu’une proportion réduite (moins de $5 \%$ de l'ensemble). Hors zone annexée, le phénomène est moins varie et nettement plus limité, quoique des pistes de recherche restent à explorer, entre autres l'internement administratif dans toute la zone sud.

L'occupation de la France et l'Annexion de fait de l'Alsace et de la Moselle par le règine par ù si un paz Vichy pétive, act 
La répression de
lhomosexualité en France entre 1940 et 1945

impact majeur immédiat, elle reste en vigueur après la Libération. Ses disposition ne sont abrogées que quarante ans après son introduction, tout en ayant constituéle ferment qui permit en 1960 le classement de l'homosexualité au rang de fléau social, également à lorigine de représailles discriminatoires. C'est dans ce contexte que vont émerger pendant les années 1970 divers mouvements d'émancipation réclamant, entre autres, l’abandon des discriminations légales. En France, c'est aussi le temps de l'émergence des revendications de reconnaissance des persécutions endurées par les homosexuels à l'époque nazie (Schlagdenhauffen, 2011, p. 148).

Par ailleurs, la stigmatisation légale après-guerre des pratiques homosexuelles (pénalisation des relations homosexuelles consenties avec les mineurs de plus de 15 ans, suivie des mesures de lutte contre l'homosexualité devenue «fléau social») n'incite pas les victimes à témoigner ouvertement de leurs épreuves lorsqu'elles ont pour origine la lutte contre l'homosexualité, qu'elle soit avérée ou supposée. Ains le seul témoin déclaré, l'Alsacien Pierre Seel, arbitrairement incarcéré, d’abord à la prison de Mulhouse, puis au camp de sécurité de Schirmeck-Vorbruck, focalise l'attention à compter des années 1980, peu après l'abandon des dernières dispositions légales discriminatoires. Erigé en symbole par les militants, il rend compte d'un vécu représ dun vécu récer a repression en Alach avec le militant Jean Le Bitoux (Seel, 1994), reste un des rares témoignages accessibles en français. Le peu de sources alors disponibles pour l'étayer, mais peut-être aussi les visées plus informatives et militantes qu'elle sert, font que cette parole recueillie plus de cinquante ans après les faits contient d'inévitables incohérences historiques

-Strafgesetzbuch mit den und den einschlägigen Bestimmungen für die neue Reichsgebiete, Berlin \& Munich, C.H. Beck'sche Verlas,

Traduction de l'auteur: En application de l'art. 3, alinéa 1, du décret du Führer du 23 mai 1940 [Renvoi au journal officiel], l'ensemble des lois du Reich ainsi que de la lé́sislation régionale de P'Etat de Prusse prendron
effet au $1^{\text {er }}$ septembre dans le secteur d'Eupen Malmedy et Moresnet. et souvenirs glanés chez d’autres témoins. L’ouvrage s'inscrit encore dans une époque où les lacunes de la recherche historique poussent les militants, confrontés au dén que leur opposent pouvoirs publics ou associations d'anciens déportés, à vouloir déceler coûte que coûte une transposition en France de la réalité répressive allemande.

La connaissance a progressé depuis et il en va de même de la reconnaissance de cette réalité historique par les institutions françaises, même s'il fallut attendre 2010 pour qu'une plaque soit apposée sur le site de l'ancien camp de concentration de Natzweiler-Struthof pour rappeler les «déportés pour motif d'homosexualité» en ces lieux. Et si aujourd'hui encore, l'attention des militants reste très axée sur le symbole du triangle rose qui traduit ce motif d'homosexualité dans le système concentrationnaire, l'étude historique ouvre de nouvelles perspectives. Elle montre en effet que cette appellation métonymique de «triangles roses» n'est plus suffisante pour couvir la diversité parmiles déportés enlien avecl'homosexulité Riensante pourcosualité. Rien été affublés de triangles verts, noirs ou rouges (respectivement pour les détenus

«droit commun», «asociaux», ou «politiques»). Certes, c'est aussi dans ce camp de concentration que l'on trouve en premier lieu les seuls Français (huit) porteurs du triangle rose, tous alsaciens ou mosellans. Pour autant, réduire la mémoire de cette répression en France aux seuls « triangles roses » reviendrait à la circonscrire à une minorité dans la minorité que représentent les quelque 30 déportés concentrationnaires en lien avec l'homosexualité (comprenant une douzaine depuis la France occupée). Accessoirement cela donnerait raison àl'ancien député Christian Vannestequi, dans des déclarations polémiques sur le sujet ${ }^{14}$ n'y voyaitjamais qu'un

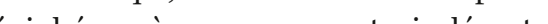
epiphenonoène propre aux trois départements annexés seulement. Mais ce serait surtout igno la varitoire français, quoiqu'il s'agisse principalement d'un phénomène affectant l'Alsace et, sans nouvelles découvertes, c'est quasi exclusivement l'homosexualité masculine qui est visée. Le devoir de mémoire, si souvent invoqué dans les cercles militants LGBT et dans les associations mémorielles, ne saurait se substituer au devoir d'histoire à l'échelle européenne.

Enfin, si l'on adopte une approche comparative, on ne peut s'empêcher de remarquer une certaine similitude entre la France et la Belgique, une fois sous contrôle de l'occupant nazi. Car la Belgique est elle aussi placé intégralement ou presque sous tutelle allemande (Commandement militaire de Bruxelles), tandis qu'un territoire-frontière, récupéré à la fin de la Première Guerre mondiale, est annexé par l’Allemagne nazie en 1940. La dernière loi annexe au Code pénal allemand dans l'édition de cette même année précise d'ailleurs qu'aux termes du décret d'Hitler daté du 23 mai, l'intégralité de la législation du Reich prendrait effet au $1^{\mathrm{er}}$ septembre dans le secteur d'Eupen, Malmedy et Moresnet. On est alors en droit de s'interroger sur la portée de ce décret pour ce qui est de la lutte contre l'homosexualité. A-t-elle été pratiquée dans cette zone d'une façon approchante à ce qui a pu être observé dans es territoires annexés français? Et qu’en est-il du reste du pays?

BIBLIOGPAPHE

- Boninchi, Marc (2005): Vichy et lordre moral, Paris, Presses

- Boulligny, Arnaud (2010) : « La déportation de France pour motif dhomosexualite, in Mickaell Bertrand (dir), La déportation pour motti
d'homosexualite en France - Débat d'histoire et enjeu de mémoire Lyon Mémoire active, p. $51-72$

- Bourgi, Hussein (2015): « Le Mémorial de la áéportation
homosexuelle », in Le collectif IDEM, Les LGBT font bouger les homosexuelle , , in Le collectif IDEM, Les LGBT for
sociétés, Paris, Des Ailes sur un Tracteur, p. 356-363. - Hoffschildt, Rainer (2013): Materialsammlung zur Geschichte
homosexueller Männer in Elsaps-Lothringen, Hanovre, Texte non publie,
- Lever, Maurice (1985) : Les Bûchers de Sodome - Histoire des Neveu, Cédric (2012) : La Gestapo en Moselle, Nancy, Serpenoise - Jackson, Julian (2009) : Arcadie - La vie homosexuelle en France, de laprese-guerre à la dépénalisation, Paris, Autrement, p. 45-55. - Schlagdenhauffen, Régis (2011): Triangle rose - La persécution des
homosexuels et sa mémoire, Paris, Autrement, p. 148. - Schwab, Jean-Luc (2015) : Mémoire de Master, Répression de

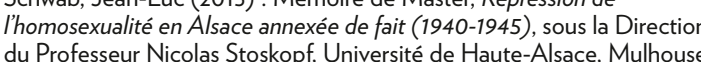
- Seel, Pierre (1994), Moi Pierre Seel, déporté homosexuel, Paris, 